EUROPEAN ORGANIZATION FOR NUCLEAR RESEARCH

European Laboratory for Particle Physics

Large Hadron Collider Project

LHC Project Report 329

\title{
HEAT FLOW MEASUREMENTS ON LHC COMPONENTS
}

\author{
D. Camacho, S. Chevassus' ${ }^{1}$, G. Ferlin, M. Pangallo' ${ }^{1}$ C. Policella, \\ J.-M. Rieubland, L. Simon and G. Vandoni
}

\begin{abstract}
The refrigeration and liquefaction capacity necessary to operate at $1.9 \mathrm{~K}$ the $27 \mathrm{~km}$ long string of superconducting magnets of the LHC has been determined on the basis of heat load estimates, including static heat inleaks from ambient temperature, resistive heating and dynamic beam-induced heat loads. At all temperature levels, the static heat inleaks determine at least one third of the total heat loads in nominal operating conditions of the machine. Design validation of individual cryocomponents therefore requires a correct estimate of the heat inleaks they induce at all temperature levels, in order not to exceed the allocated heat budget. This paper illustrates the measurements of heat inleaks for several cold components of the future machine, including insulating supports, radiation shields, multi-layer insulation, instrumentation current leads. Distinct methods to determine the heat flow are chosen, depending on the expected heat loads, the temperature range spanned by the heat intercepts, and the working conditions of the component itself.
\end{abstract}

LHC Division, CERN, 1211 Geneva 23, Switzerland

1 SGG EEIG/ AMSE - Technoparc Gessien - 01630 St.Genis-Pouilly

Presented at the 1999 Cryogenic Engineering and International Cryogenic Materials Conference

(CEC-ICMC'99), 12-16 July 1999, Montreal, Canada

\footnotetext{
Administrative Secretariat

LHC Division

CERN

CH - 1211 Geneva 23

Switzerland

Geneva, 1 December 1999
} 


\title{
HEAT FLOW MEASUREMENTS ON LHC COMPONENTS
}

\author{
D.Camacho, S.Chevassus ${ }^{1}$, G.Ferlin, M.Pangallo ${ }^{1}$, C.Policella, \\ J.-M.Rieubland, L.Simon, G.Vandoni \\ CERN, European Organization For Nuclear Research, 1211 Geneva 23, \\ Switzerland \\ ${ }^{1}$ SGG EEIG/ AMSE - Technoparc Gessien - 01630 St.Genis-Pouilly
}

\begin{abstract}
The refrigeration and liquefaction capacity necessary to operate at $1.9 \mathrm{~K}$ the $27 \mathrm{~km}$ long string of superconducting magnets of the LHC has been determined on the basis of heat load estimates, including static heat inleaks from ambient temperature, resistive heating and dynamic beam-induced heat loads. At all temperature levels, the static heat inleaks determine at least one third of the total heat loads in nominal operating conditions of the machine. Design validation of individual cryocomponents therefore requires a correct estimate of the heat inleaks they induce at all temperature levels, in order not to exceed the allocated heat budget. This paper illustrates the measurements of heat inleaks for several cold components of the future machine, including insulating supports, radiation shields, multi-layer insulation, instrumentation current leads. Distinct methods to determine the heat flow are chosen, depending on the expected heat loads, the temperature range spanned by the heat intercepts, and the working conditions of the component itself.
\end{abstract}

\section{INTRODUCTION}

The Large Hadron Collider (LHC), CERN's next generation particle accelerator, uses superconducting magnets operating in pressurised helium II at $1.9 \mathrm{~K}$ and $0.1 \mathrm{MPa}$ for bending and focusing the particle beams. The thermodynamic cost of refrigeration below the superfluid transition temperature $T_{\lambda}$ requires that heat loads at this temperature level be minimised, by effective insulation, multiple temperature staging and careful thermal design of components ${ }^{1}$. Calculation alone is often insufficient to evaluate the thermal load from a complex component where all thermal transfer mechanisms might be acting, so design validation is incomplete without testing. Accurate measurement of the heat flow in a complex component through radiation, conduction, convection, demands that its working environment in operating conditions be closely reproduced in the test-bench. Conversely, the component has to be isolated with respect to the cryostat, so that only its own heat loads are measured. This necessity rules out the method by vaporisation of the liquid helium bath, which sums all the heat inleaks coming from the cryostat. In this paper, we present other, more accurate possibilities, and show some results obtained by applying them. 


\section{HEAT LOADS ABOVE $T_{\lambda}$}

Radiative and conductive losses of an anticryostat. The instrumentation applied to perform magnetic measurements on the $15 \mathrm{~m}$ long superconducting dipole magnets, operates at $300 \mathrm{~K}$ inside the cold bore $(1.8 \mathrm{~K})$ of the magnets. The cylindrical anticryostat ${ }^{2}$ yields the major component of the heat loads on the cryogenic magnet test benches. In the tight space between cold and warm bore $(5 \mathrm{~mm})$, insulation is achieved using six layers of MLI, supported on the warm bore and held together by a floating radiation shield, onto which are mounted insulating supports in polyimide. The ensemble has to assure the mechanical support for the measuring instrumentation and the isothermal conditions of the measurement. The scope of the measurement presented here was to comparatively assess the effectiveness of an aluminium coating on the radiation shield upon the insulating performance of the anticryostat. Evaluation of the heat losses is obtained by internally heating a short segment of the anticryostat to bring it to steady-state, so that the electrical heating balances the heat losses to the surrounding bath at $4.2 \mathrm{~K}$.

The set-up is displayed on Figure 1. In order to limit the effect of fringe losses from the end parts, two heated guard shields screen the tube from ambient and from the bottom of the cold bore. Their temperature is regulated to be equal to that of the warm bore. The vacuum space between the warm and the cold bore is pumped both mechanically and by active charcoal. The anticryostat is suspended inside the cold bore with minimal thermal contact to it, ensured by conical fibreglass tips resting on the base surface.

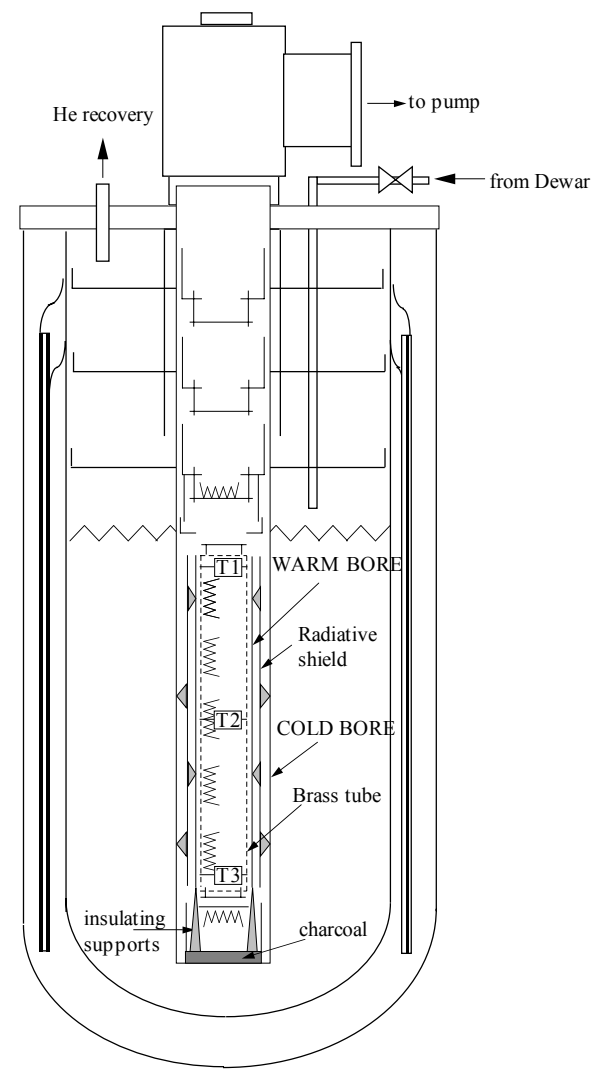

Figure 1: Schematic view of the test-bench for the heat inleak measurements from the anticryostat. A tube containing the anticryostat segment is immersed into a cryostat containing liquid helium at 4.2 K. The anticryostat is heated internally to a constant temperature, the heating power balancing the losses to the surrounding helium bath. 
By varying the steady-state temperature, it is possible to separate the contribution of radiation and conduction from the polyimide supports by their different temperature dependence. Steady-state heat flow versus temperature for both the anticryostat with aluminised and with polished steel radiation shield is plotted on Figure 2.

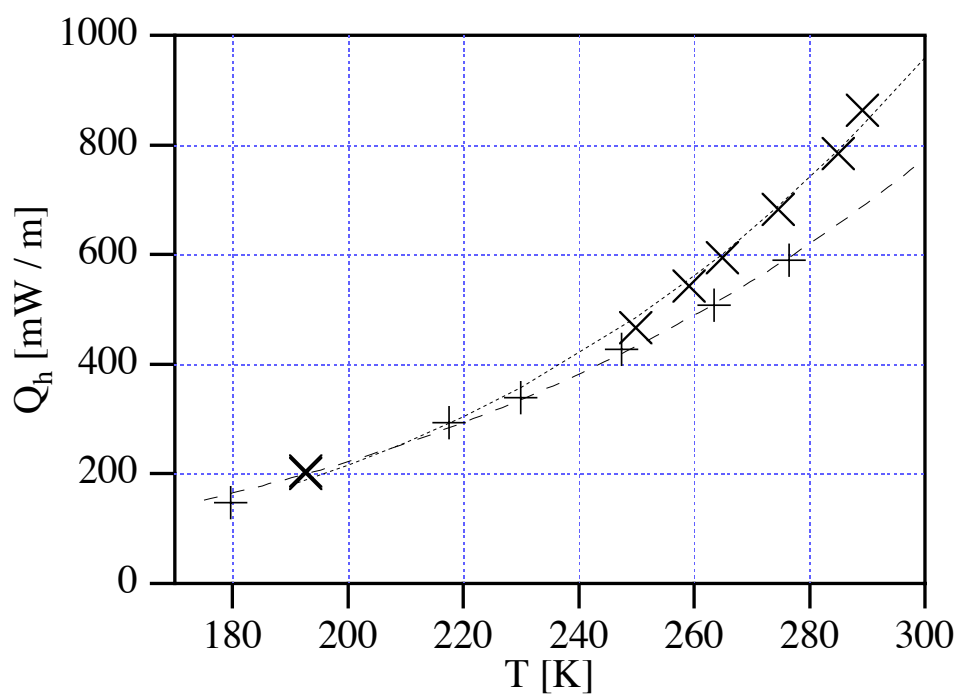

Figure 2: Measured heat losses per unit length of the anticryostat with a radiation shield in aluminized steel $(+)$ and simply polished steel $(X)$, as a function of equilibrium temperature.

The data are fitted to a polynomial of the form $Q=A T+B T^{4}$, in which the first term accounts for conduction and the second one for radiation. A linear extrapolation permits us to infer separately the coefficients A and B. Evaluation of the separate contribution of radiation and conduction at $300 \mathrm{~K}$ in the two cases is illustrated in Table 1 . The estimated error of $\sim 5 \%$ on the global losses results from the calculation of residual heat flux to and from the cryostat.

Table 1: Conduction and radiation losses from the anticryostat

\begin{tabular}{l|l|l|l}
\hline Anticryostat with & Conduction $[\mathrm{mW} / \mathrm{m}]$ & Radiation $[\mathrm{mW} / \mathrm{m}]$ & Total losses [mW/m] \\
\hline Al-coated screen & 279 & 497 & $776 \pm 39$ \\
\hline Polished steel screen & 105 & 854 & $959 \pm 48$ \\
\hline
\end{tabular}

Thermal performance of MLI So called heatmeters ${ }^{3,4}$ constitute today a frequently applied method for the measurement of heat loads, excluding any interference by the residual losses of the test bench. With respect to other techniques, they present the advantage of being designed to yield the desired heat flow range and/or sensitivity. Briefly, a heatmeter is a calibrated thermal impedance, which permits the user to infer the heat flow by the measurement of the temperatures at its extremities. Since it is mounted between the component under test and its heat sink, it presents the obvious disadvantage of introducing a major perturbation of the system, hence its impedance is determined by a trade-off between sensitivity and maximum allowable temperature difference between the component and the heat sink.

An example of the use of heatmeters is presented at this conference ${ }^{5}$, where the thermal design of a dipole support in composite material is qualified experimentally. Here, we present an application of heatmeters in the evaluation of the heat losses from multi-layer insulation (MLI), operating between $77 \mathrm{~K}$ and $4 \mathrm{~K}$.

A schematic view of the test-bench is presented in Figure 3. The MLI blanket is tested in a horizontal configuration. The main drawback of this configuration is the presence of a 
circular edge, exchanging heat with the environment. On the other hand, provided that the plates can be modelled as infinite flat parallel surfaces, the heat exchanged by radiation between them is independent of their surface area. This is not the case for concentric cylinders, since here the heat flow depends on the ratio between the surface areas.

Hence, if the edge effect is minimised, we believe that a measurement in a flat configuration is preferable to one in a cylindrical configuration to compare the performance of $\mathrm{MLI}^{6}$

A $600 \mathrm{~mm}$ diameter MLI sample is placed on an aluminium supporting disk, the captor plate, below which the heatmeter is mounted. The emitter plate faces the captor with as small as possible a distance between them. In order to limit the error induced by the finite geometry with respect to the infinite one, a mirror ring guard is mounted around the border of the warm emitter plate. The role of this guard is to reflect back onto the captor plate the radiation which escapes the space between the two plates by multiple reflections.

Regulation of the emitter plate temperature is achieved by electrical heating and by conduction cooling through a thermal anchor to a radiation screen. Two concentric screens ensure the insulation of the test chamber from radiation from the vacuum vessel walls. The emitter plate is connected to the cold extremity of the heatmeter, thus avoiding any conduction heat leak between emitter and captor.

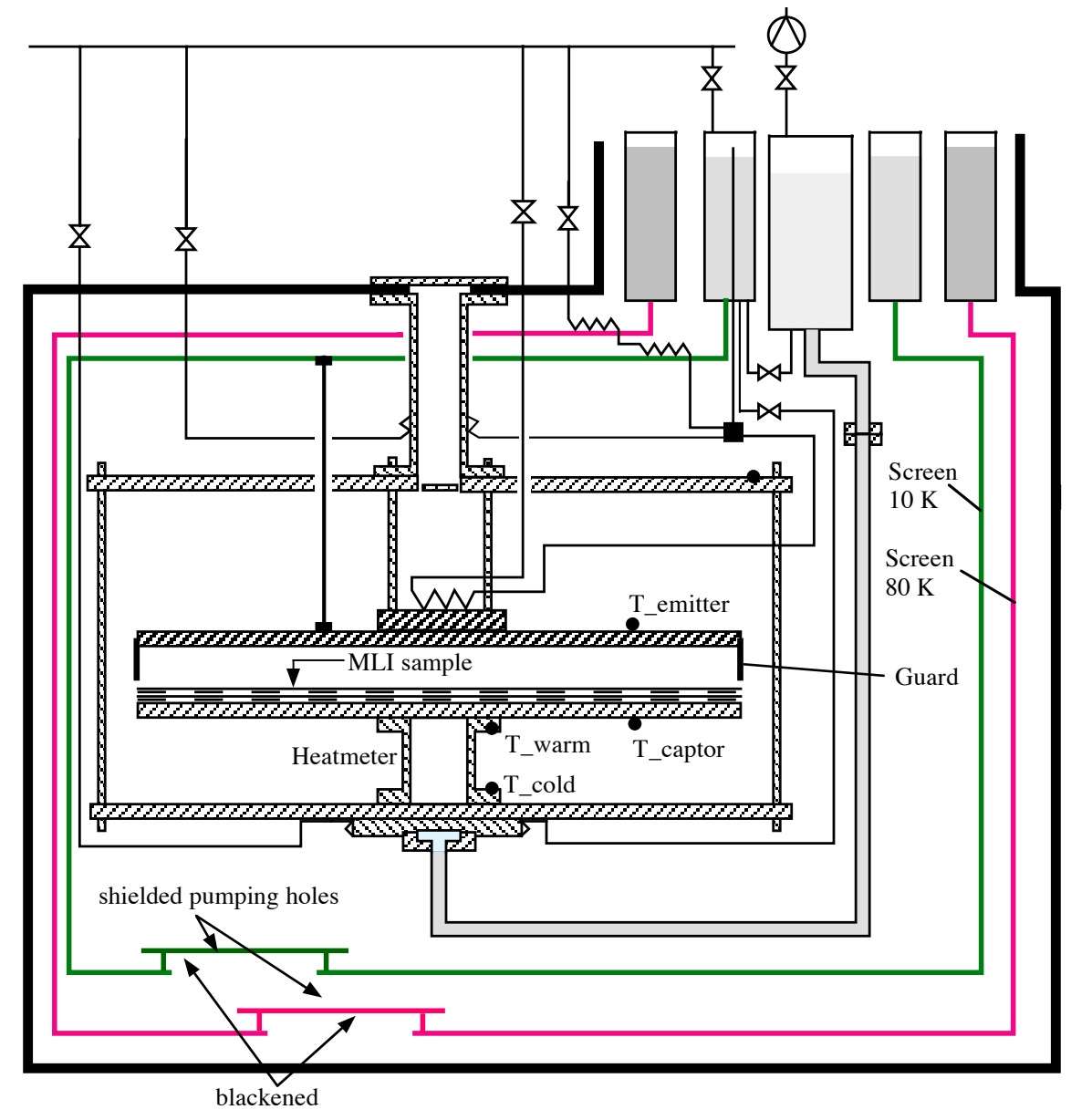

Figure 3: Test-bench for MLI tests in a horizontal geometry.

Owing to the large surface of the captor plate, the smallest residual radiation flux from the environment will induce an error on the measured heat flow. In order to evaluate this background effect, a black captor is used: this is realised with an anodised aluminium disk, whose emissivity at low temperature exceeds 0.8 . Keeping the emitter plate at the same cold 
temperature as the captor, we can then measure the heat captured by the black plate from the environment.

In a first design of the test-bench, the pumping holes on the bottom of the radiation screens were shielded with aluminium disks. The background radiation measured by the black plate amounted to $32 \mathrm{~mW} / \mathrm{m}^{2}$. In an effort to reduce this background, the outer face of these disks was blackened by anodization. This reduced the background heat load to the black plate to $10 \mathrm{~mW} / \mathrm{m}^{2}$. Subsequent measurements were always preceded by an evaluation of the background heat load, keeping the emitter plate cold and measuring the heat flow to the heatmeter. Provided the temperature of the internal radiation screen is kept below $10 \mathrm{~K}$, this effect is limited to $\sim 1 \mathrm{~mW} / \mathrm{m}^{2}$.

The main challenge of a horizontal set-up for qualifying MLI is constituted by the minimisation of edge effects. It is easy to estimate an upper limit for the effect of the free edge of the MLI disk. Assuming a perfect system of $\mathrm{N}$ floating radiation screens, the temperature of the $\mathrm{i}$-th screen is given by:

$$
T_{i}=\sqrt[4]{T_{w}^{4}-\frac{i}{N+1}\left(T_{w}^{4}-T_{c}^{4}\right)}
$$

The edge space between two layers $i$ and $i+1$ can be seen as a black ring, radiating with temperature $\left(T_{i}+T_{i+1}\right) / 2$ on the aluminium guard screen, held at $10 \mathrm{~K}$. Calculation of the total heat lost by radiation from the MLI to the cold screen yields $2 \mathrm{~mW}$ per meter length of the edge. In reality, owing to a conductive contribution to the heat flow between the screens, the $\mathrm{i}$-th screen temperature will be lower than the value of $T_{i}$ given by eq.(1), hence an estimation of edge effects based on this temperature profile can be considered as pessimistic.

The test-bench has been applied to the evaluation of the effect on the thermal performance of MLI of an insulating fibreglas net, placed on top of the first layer to increase its rigidity and facilitate fitting of the blanket. The MLI blanket is made up by 10 spacers and 10 double-aluminised reflectors. Measurements are performed on the bare blanket, and on the blanket covered by the insulating net. A purely geometrical estimation yields a coverage by this net of approximately $36 \%$ of the surface.

In order to increase the precision of the measurement, the test was done at variable emitter temperature.

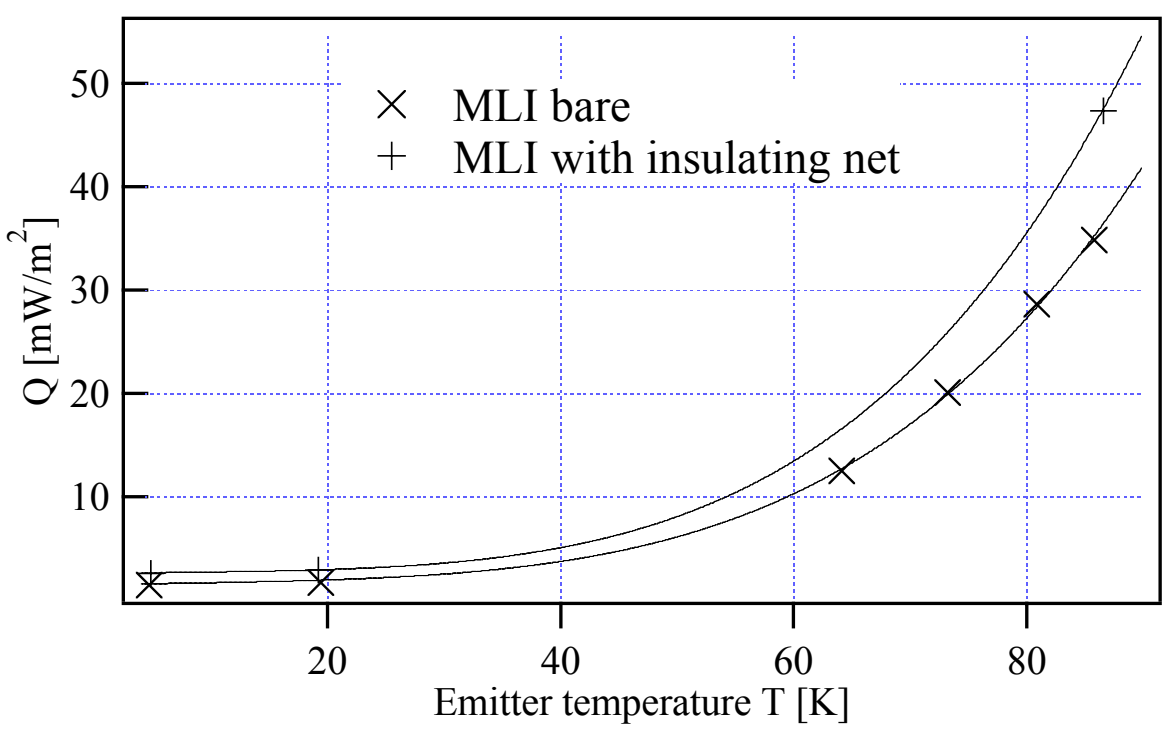

Figure 4: Heat flux to $4 \mathrm{~K}$ through an MLI blanket as a function of emitter temperature from a simple blanket, in one case, or with the blanket covered with an insulating net. The area of the captor amounts to $0.28 \mathrm{~m}^{2}$.

Figure 4 displays measured data points, fitted with a polynomial of the form $\dot{Q}=A T+B T^{4}$. Similar equations are often applied to describe the thermal performance of $\mathrm{MLI}^{7}$. The fit 
permits an estimation of the heat flow for a warm-boundary temperature of exactly $80 \mathrm{~K}$ with and without fiberglass net (see Table 2). It appears that the insulating net increases the heat flux between $80 \mathrm{~K}$ and $4 \mathrm{~K}$ by as much as $30 \%$.

Table 2: Thermal performance of MLI with/without insulating net

\begin{tabular}{|c|c|c|}
\hline MLI type & Heat flow from $80 \mathrm{~K}$ to $4 \mathrm{~K}$ & Heat flux \\
\hline 10 reflectors +10 spacers & $7.6[\mathrm{~mW}]$ & $27.0[\mathrm{~mW} / \mathrm{m} 2]$ \\
\hline \multirow[t]{2}{*}{$\begin{array}{l}10 \text { reflectors }+10 \text { spacers } \\
\text { with fibreglas net }\end{array}$} & $10.0[\mathrm{~mW}]$ & $35.4[\mathrm{~mW} / \mathrm{m} 2]$ \\
\hline & $\%$ Difference & $+30 \%$ \\
\hline
\end{tabular}

\section{HEAT LOADS BELOW $\mathrm{T}_{\lambda}$}

An instrumentation feedthrough with open end. An effect which is sizeable only for superfluid helium, the Kapitza resistance to heat transfer at the interface between a solid and the superfluid can be exploited to realise a highly sensitive heatmeter below $\mathrm{T}_{\lambda}{ }^{8}$. The heat leak of components working with their cold end in a superfluid helium bath can be measured by the temperature difference generated across a copper disk separating a pressurised HeII cell, into which terminates the component, from a saturated HeII heat sink. The Kapitza conductance depends on pressure through the density of the liquid, hence the error induced by measuring at 1 bar instead than at saturation pressure is only of the order of $1 \%$. Conversely, the strong temperature dependence of the Kapitza conductance imposes careful calibration of the device.

Assuming that the temperature drop through the copper disk of area $\mathrm{S}$ is negligible, the heat transfer across the two surfaces separating two superfluid baths is equal to

$$
\dot{Q}=\dot{Q}_{h}+\dot{Q}_{o}=C_{K} S \frac{\Delta T}{\frac{1}{T_{1}^{3}}+\frac{1}{T_{2}^{3}}}
$$

with $C_{K}$ a material characteristic, $Q_{o}$ the residual heat flow from the environment, and $Q_{h}$ either the heat flow from the component, or the one applied electrically to a calibration heater. Keeping the temperature of the "warm" bath, $T_{2}$, constant, and applying to it a known heating power $Q_{h}$, we obtain calibration curves like the ones displayed on Figure 5. From these curves we can easily extract the value of $C_{K}$ and the residual heat inleak $Q_{o}$ to the warm bath.

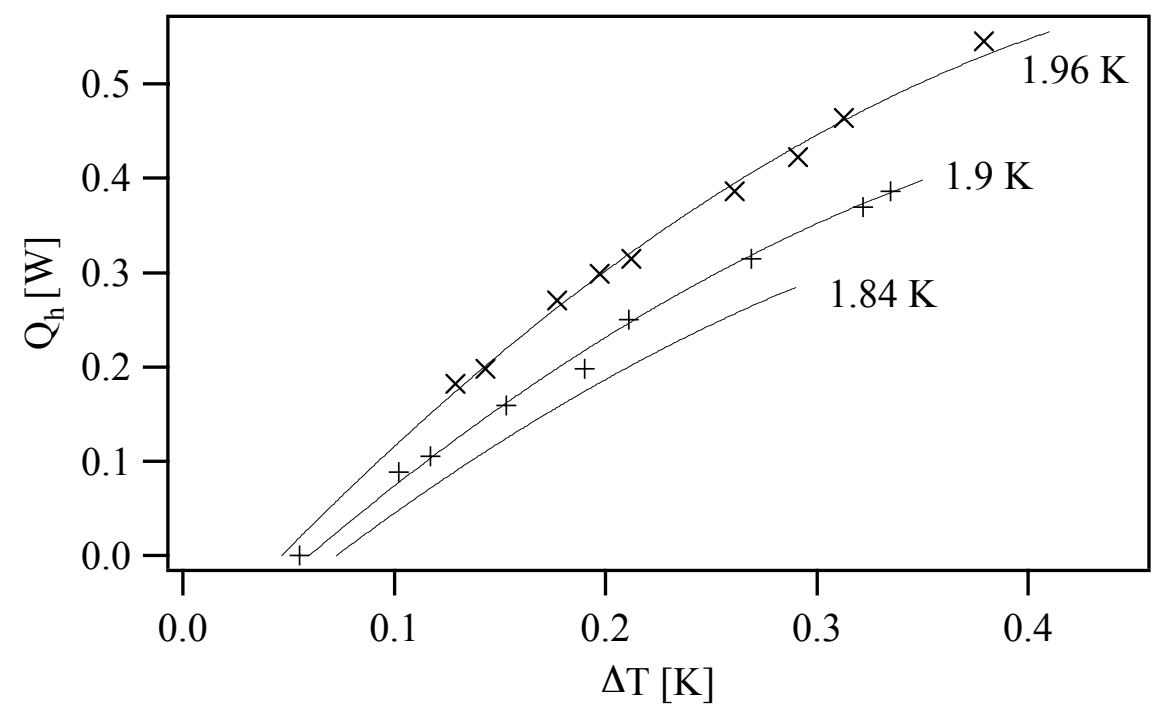

Figure 5: Calibration of a Kapitza heatmeter at variable temperature. 
Since $C_{K}$ depends on the surface cleanliness, some variation in time can be observed, requiring regular re-calibration of the system. A regulation system on the pumping of the saturated HeII bath can be used to keep the "warm" bath temperature constant. In this case, $Q_{o}$ remains constant, and the simple reading of $T_{1}$ is sufficient to extract the heat load of the component from the corresponding measured calibration curve. Even more simply, the electrical resistance of the temperature sensor can be used to construct a calibration curve $Q=Q\left(R_{1}\right)$, if $T_{2}$ is kept constant. Calibration can also be re-checked with the tested component in-situ, by applying increasing steps of heating power to the warm bath, still keeping its temperature constant.

The area of the copper disk is adapted to the expected heat flow, so that the device ensures a good sensitivity, still permitting not to exceed the superfluid transition on the warm side. Different components have been measured by applying a Kapitza heatmeter ${ }^{8}$. In this conference 9 , a paper describes a test facility for the characterisation of safety relief valves based on the same device. We focus here on measurements devoted to thermally characterise an open-end capillary feedthrough for cold instrumentation wire routing from the LHC magnets to ambient. The thermal leak through such a tube depends both on the solid conduction through the wires and on the conduction through the superfluid helium column inside the tube.

Figure 6 illustrates the scheme of the test-bench. The model capillary consists of a 2.3 $\mathrm{m}$ long corrugated stainless steel tube, of smallest inner diameter $7.7 \mathrm{~mm}$, with smooth terminations. On the cold side, the ending tube has an inner diameter of $8 \mathrm{~mm}$. The tube contains 40 insulated copper wires, of cross section $0.14 \mathrm{~mm}^{2}$. The warm termination of the capillary is linked to a pressure gauge, the wires are connected to the vessel at ambient temperature. Two cylindrical screens surround the capillary, each spanning approximately one third of the total length. The screens are cooled by separate He gas flows, and equipped with a resistive heater, to control their temperature. The cold termination of the capillary is connected to a pressurised HeII cell at $1.9 \mathrm{~K}$, which constitutes the warm end of a Kapitza heatmeter.

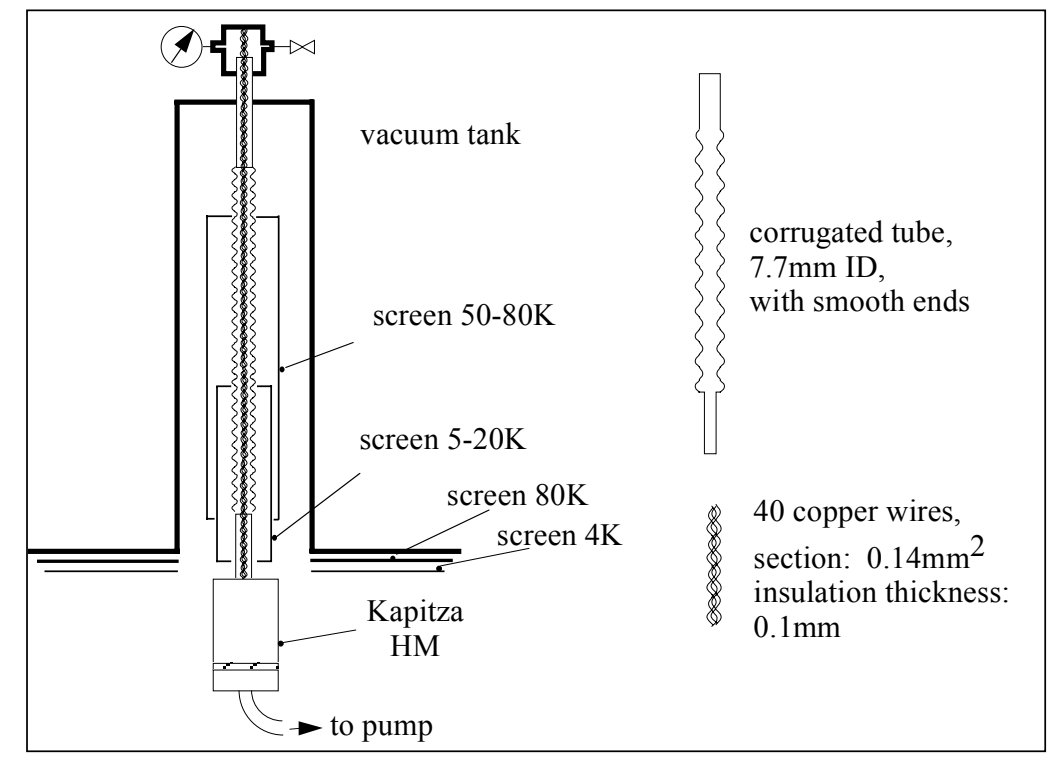

Figure 6: Schematic set-up for the measurements of heat losses from an instrumentation feedthrough.

The measurement results are displayed on Table 3. In order to evaluate the effect of radiative heat load onto the walls of the capillary, after the first set of measurements the segment of capillary seeing the warm screen has been covered by a layer of aluminized mylar, over one third of the total length. At identical temperature conditions, the heat flow to the HeII bath is thus reduced by $40 \mathrm{~mW}$. Notice that the error bars given in the table indicate absolute precision, whereas reproducibility amounts to $1 \%$. 
Table 3: Heat losses from an instrumentation feedthrough

\begin{tabular}{l|l|l|l|l}
\hline & T_cold [K] & T lower screen $[\mathrm{K}]$ & T upper screen $[\mathrm{K}]$ & $\mathrm{Q}[\mathrm{mW}]$ \\
\hline Unshielded tube & $1.92 \pm 0.01$ & $4.5 \pm 0.05$ & $63.5 \pm 0.5$ & $544 \pm 25$ \\
\hline Shielded tube & $1.92 \pm 0.01$ & $4.6 \pm 0.05$ & $62.1 \pm 0.8$ & $504 \pm 25$ \\
\hline
\end{tabular}

The heat flow through the open capillary can be roughly estimated by assuming that all the heat is carried by conduction through the copper wires above the level where the superfluid transition takes place, and by conduction through the superfluid column below this level. The height of the superfluid column inside the capillary is obtained equalising these two heat flows, and taking the variable section geometry into account. The calculation yields a heat flow of $525 \mathrm{~mW}$, in fairly good agreement with the measurement, and a superfluid column height of approximately $20 \mathrm{~cm}$.

\section{CONCLUSION}

Precise measurements of heat loads from complex components require at the same time a complete control of the residual heat inleaks from the cryostat and a set-up which closely reproduces the working environment of the component. Here, we have applied different methods, like calorimetry, solid and Kapitza heatmeters, to the measurement of the thermal losses of an anticryostat, to the thermal evaluation of MLI, and to the test of an open-end instrumentation capillary.

\section{ACKNOWLEDGEMENTS}

The work of D. Camacho is supported by the Spanish Ministry of Education and Culture under the Subprograma de Especializacion en el Laboratorio Europeo de Fisica de Particulas

\section{REFERENCES}

1. P. Lebrun, "Advances in Cryogenics for the Large Hadron Collider" LHC-Project-Report 211, $17^{\text {th }}$ International Cryogenic Engineering Conference, Bournemouth UK, 14-17July, 1998 and references herein. 2. F. Clari, O. Dunkel, M. Genet, Ch. Gregory, P. Sievers, 1994. 13th International Conference on Magnet Technology. Victoria, B.C., Canada; 20-24 Sept.1993. Publ. in: Proceedings R B Goldfarb IEEE Trans. Magn.: 30 (1994) (2662-2664)

3. H. Danielsson, P. Lebrun and J.-M. Rieubland, "Precision Heat Inleak Measurements on Cryogenic Components at 80K, 4.2K and 1.8K" Cryogenics Vol.32 ICEC Supplement, p.215 (1992)

4. G. Ferlin, B. Jenninger, J.-M. Rieubland, "Precise wide range heatmeters for $1.5 \mathrm{~K}$ up to $80 \mathrm{~K}$ ", Advances in Cryogenic Engineering Vol.43, p.811

5. M. Castoldi, M. Pangallo, V. Parma, G. Vandoni, "The Thermal Performance of the Supporting System for the Large Hadron Collider (LHC) Super-conducting Magnets", (Abstract \#COA-5) CEC/ICMC, July 12-16 1999, Montreal

6. Handbook of Cryogenic Engineering, J.G. Weisend II editor, Taylor \& Francis, Philadelphia, Ch. 3-4-1 7. C.W. Keller, G.R. Cunnington, and A.P. Glassford, "Thermal Performance of Multilayer Insulations", NASA report, CR-134477 (1974)

8. H. Danielsson, G. Ferlin, B. Jenninger, C. Luguet, S-E. Milner, J.-M. Rieubland, "Cryogenic Performance of a Superfluid Helium Relief Valve for the LHC Superconducting Magnets", CERN AT/95-43 and Advances in Cryogenic Engineering Vol. 41.

9. A. Bezaguet, L. Dufay, G. Ferlin, A. Perin, G. Vandoni, , “A Facility for Accurate Heat Load and Mass Leak Measurements on Superfluid Helium Valves”, (Abstract \#CDD-4) CEC/ICMC, July 12-16 1999, Montreal 